\title{
Influence of Ethanol-ferric Chloride Pretreatment on Straw Enzymatic Saccharification
}

\author{
Wei Chen \\ China Merchants Chongqing Communications Technology Research \& Design Institute Co. LTD \\ cqchenwei@cmhk.com
}

\begin{abstract}
Keywords: Ethanol-ferric chloride; Pretreatment; Straw; Enzymatic saccharification.
Abstract. Since sufficient raw material supply and renewable energy development demand are available, fuel ethanol made of lignocellulose has very broad prospects for development. In the paper, straw is regarded as research object to study the influence factor of organic solvent pretreatment on straw enzymatic saccharification. Influence on enzymatic saccharification after straw pretreatment and improvement of cellulose conversion efficiency in straw through pretreatment are studied mainly aiming at organic solvent pretreatment temperature, time and organic solvent content as set factors. The results show that circulation chain of pretreatment, recycling and saccharification fermentation supplementing can be formed by organic solvent treatment method of replacing acetone with ethanol. After ethanol-ferric chloride organic solvent is adopted for pre-treating straw, straw enzymatic saccharification rate can be improved, and pretreatment time is decreased with the increase of temperature.
\end{abstract}

\section{Introduction}

The demand for energy is increasing constantly with sustained and rapid development of economy and society. Subsequent problem has become a major obstacle limiting economic and social development, such as energy deficiency, environmental pollution, etc. Therefore, development of renewable and pollution-free new energy has become an important research topic. All countries are widely interested in biological fuel ethanol as a renewable and low pollution new energy. Preparation of ethanol with lignocellulose as raw materials is recognized all over the world generally. Lignocellulose has characteristics of wide sources, low cost, high conversion rate, etc. Rice is a grain crop with large-area planting in China. Hundreds of millions of tons of straw are produced each year [1-3]. Meanwhile, straw also can be used as an important biological resource with practical significance. Because preparation of ethanol by glucose fermentation has formed a mature industrial production process [4-5], pretreatment and saccharification stages are mainly studied in the paper. Straw is selected as research object in the paper, acetone, ethanol and other organic solvents are added with phosphate for mixed pretreatment. Then, cellulose is added for enzyme saccharification, thereby converting straw cellulose into glucose.

\section{Measurement method and experiment principle}

Determination of glucose. Glucose of more than $2 \mathrm{~g}$ is weighed and placed in electrothermal constant temperature oven, which is dried at 98DEG C to constant weight (24h). Glucose $0.1 \mathrm{~g}$ is weighed accurately, which is dissolved in distilled water, the volume is fixed constantly to $100 \mathrm{ml}$. A number of $10 \mathrm{ml}$ colorimetric tubes are configured and operated according to Table 2.1. They are mixed fully and heated for $5 \mathrm{~min}$ in boiling water then. The volume is fixed to $10 \mathrm{ml}$ after cooling. Spectrophotometer is used for measuring absorbance after being placed for 20min (detection wavelength of 550nm). Glucose standard curves are obtained.

Table 1 Preparation of glucose standard solution

\begin{tabular}{cccc}
\hline No. & Glucose $(\mathrm{ml})$ & Distilled water $(\mathrm{ml})$ & DNS(ml) \\
\hline 1 & 0 & 0.5 & 1.5 \\
2 & 0.1 & 0.4 & 1.5 \\
3 & 0.2 & 0.3 & 1.5 \\
\hline
\end{tabular}




\begin{tabular}{lccc}
\hline 4 & 0.3 & 0.2 & 1.5 \\
5 & 0.4 & 0.1 & 1.5 \\
6 & 0.5 & 0 & 1.5 \\
\hline
\end{tabular}

Enzymolysis reaction liquid $0.5 \mathrm{ml}$ is taken, 3.5- DNS $1.5 \mathrm{ml}$ is added, and the method of determining glucose standard solution is used for determining glucose concentration in enzymolysis liquid. (Since absorbance exceeds standard curve absorbance sometimes during determination of glucose concentration in enzymolysis liquid, the liquid should be diluted for determination aiming at determination of the part).

Determination of ash content in straw. In the experiment, ash content in straw is determined by infrared rapid coal quality analyzer produced by Changsha Kaiyuan Instrument Co., Ltd. Thermogravimetric analysis method is the detection principle of instrument. Infrared heating equipment is combined with weighing electronic balance. The specimens in the heated process are weighed under certain atmosphere conditions, regulated temperature and regulated time, therefore specimen ash content and other industry analysis indicators are calculated as a result.

Specific testing process is shown as follows: instrument test procedure is operated, work test menu is started, empty crucible weight can be weighed automatically by the instrument after related information is input, the system prompts the user to place analysis coal samples $(<1 \mathrm{~g})$, and then the system starts to weigh the sample weight and heat high temperature furnace (oxygen valve is opened by the system, the flow meter on the panel is adjusted, and gas flow is controlled in $3 \sim 4 \mathrm{~L} / \mathrm{min}$ ). High temperature furnace is heated to 500DEG C, the temperature is kept constant for 30 minutes, which is then heated to 815DEG C temperature and kept constant for 30 minutes. Then, the system starts to weigh the crucible. Ash content analysis is ended when crucible weight change is less than the system set value (default value of $0.0005 \mathrm{~g}$ ). The ash content determination results are reported by the system. Calculation of cellulose saccharification rate in straw. Straw enzymolysis after pretreatment is timed since reaction respectively at $0 \mathrm{~h}, 4 \mathrm{~h}, 8 \mathrm{~h}, 12 \mathrm{~h}, 24 \mathrm{~h}, 36 \mathrm{~h}, 48 \mathrm{~h}, 72 \mathrm{~h}$ and $96 \mathrm{~h}$ after reaction. $1 \mathrm{ml}$ transfer pipette is used for taking enzymolysis fluid. The concentration of glucose is determined. Cellulose saccharification rate of each time point can be calculated according to the following formula.

\section{Saccharification rate $=\underline{\text { Generated glucose weight }(\mathrm{g}) * 0.9 * 100 \%}$ \\ Cellulose weight in straw (g)}

Experiment principle. Catalyst in the chemical reaction has the functions of reducing reaction activation energy peak value and improving the reaction rate. Even when lignin is removed from organic solvent under the acidic effect, effective fracture of lignin molecules and soluble ingredients depends on proton catalysis [6]. Lignin removal under proton catalysis is different from dissolution of lignin with organic solvent only. Lignin is removed through proton catalysis due to hydrolysis of alpha and beta- aryl-ether bands in lignin molecules. When organic solvent is used for removing lignin in papermaking pulping process, the applied proton catalysts are divided into alkaline earth metal salts, soluble magnesium salt, soluble calcium salt and ferric chloride [7].

Holtzapple, etc. [8] used ethanol solvent for pretreatment of poplar. When saccharification rate enzymolysized by cellulose enzyme is improved, aluminum sulphate and aluminum chloride are used for catalysis. Liu, etc [9] adopt ferric chloride for pretreatment of corn stalks, the digestion rate can reach 98\% under the condition of 160DEG C and 20min. Because ferric chloride pretreatment can decompose hemicellulose easily and effectively and convert them into polysaccharides and monosaccharide, ferric chloride can be adopted for replacing phosphoric acid in decomposing hemicellulose. In addition, ferric chloride can play a promoting effect to lignin removal as proton catalyst. Therefore, room temperature and pressure organic solvent of ethanol-ferric chloride is used for pre-treating straw in the paper. The influence of time and temperature on straw enzymatic saccharification in the pretreatment process is explored. 


\section{Experimental materials and methods}

Experimental materials. Straw collected from Chongqing suburb rural areas is adopted as saccharification raw material. The physical and chemical indicators are shown in table 3.1. Straws are sheared and crushed artificially. 40-mesh sieve and 80-mesh sieve are used for screening respectively. Straw with particle size of $0.2 \mathrm{~mm}$ to $0.45 \mathrm{~mm}$ is retained as experimental sample.

Pretreatment of straw. Straw $0.5 \mathrm{~g}(0.2 \mathrm{~mm} \leqslant$ particle size $\leqslant 0.45 \mathrm{~mm})$ is weighed and placed in a $150 \mathrm{~mL}$ conical flask. Mixed pretreatment liquid $50 \mathrm{ml}$ is made of ethanol $25 \mathrm{ml}$ and $0.1 \mathrm{~mol} / \mathrm{L}$ ferric chloride $25 \mathrm{ml}$. It is heated by constant temperature water bath, and the temperature is kept. Pretreatment time and temperature are regarded as set factors respectively for studying the influence of all factors on straw saccharification after pretreatment. Design of experiment is shown in table 5.1.

Straw after pretreatment is filtered by funnel and filter paper, thereby collecting filtrate. Then, boiling water and distilled water at room temperature are used for washing the straw separately (repeated for three times). After the final washing is completed, the filtrate is placed for $3 \sim 5 \mathrm{~h}$, straw does not have prominent water content.

Table 2 Experiment design of rice straw pretreated with ethanol- $\mathrm{FeCl}_{3}$

\begin{tabular}{ccc}
\hline No. & Pretreatment time & Pretreatment temperature \\
\hline 1 & $24 \mathrm{~h}$ & Room temperature \\
2 & $48 \mathrm{~h}$ & Room temperature \\
3 & $72 \mathrm{~h}$ & Room temperature \\
4 & $1 \mathrm{~h}$ & 50DEG C \\
5 & $1 \mathrm{~h}$ & 60DEG C \\
6 & $1 \mathrm{~h}$ & 70DEG C \\
7 & 0 & $/$ \\
\hline
\end{tabular}

Straw enzymatic saccharification

Straw after pretreatment, washing and placement is transferred into $150 \mathrm{ml}$ conical flask. Then cellulose enzyme $2 \mathrm{mg}(\mathrm{IU} \geqslant 15000)$ and $0.05 \mathrm{~mol} / \mathrm{L}$ HAC-NaAC buffer $(\mathrm{pH}=5) 50 \mathrm{ml}$ are added for enzymatic hydrolysis under the condition of 50DEG C constant temperature water bath heating.

\section{Results and discussion}

\section{Comparison of straw enzymatic saccharification after pretreatment of different organic solvents}

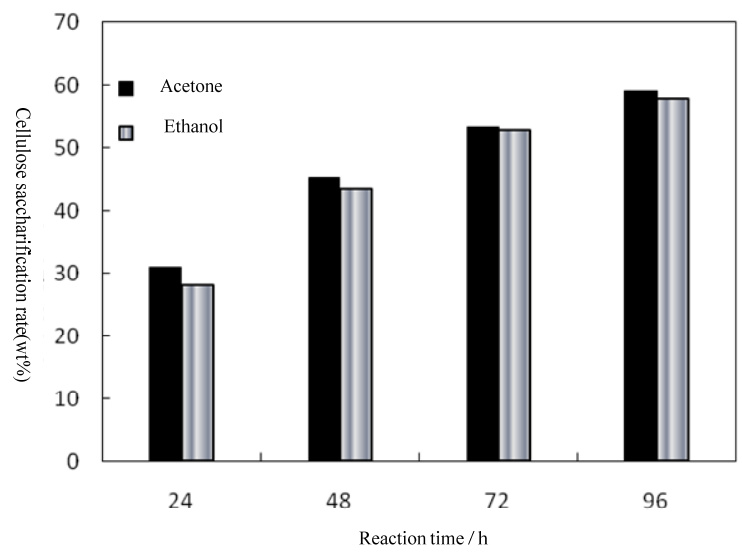

Figure 1 The effect of different organosolv pretreatment on rice straw saccharification Figure 1 shows changes of cellulose saccharification rate in straw after pretreatment of different organic solvents. The figure shows that $24 \mathrm{~h}$ acetone treatment saccharification rate is $30.97 \%$, ethanol treatment saccharification rate is 28.04; acetone treatment saccharification rate is $53.25 \%$, ethanol treatment saccharification rate is $52.84 \%$ after $72 \mathrm{~h}$; when the liquid is reacted for $96 \mathrm{~h}$, acetone treatment saccharification rate is $58.9 \%$, and ethanol treatment saccharification rate is $57.75 \%$. Straw saccharification rate after acetone pretreatment is higher than ethanol pretreatment 
slightly possibly because acetone ability in dissolving lignin is stronger than ethanol and more lignin can be removed. Different scholars have different views aiming at influence of lignin removal on cellulose saccharification. When Teramoto, etc. [10] study pretreatment influence on woody plants, it is found that cellulose saccharification rate is increased with removal of lignin. However, when a large number of lignin is removed, it does not lead to higher cellulose saccharification rate. Araque, etc. [11] study and discover that cellulose enzymolysis does not have prominent correlation with lignin content after pretreatment. Lignocellulose crystallinity and lignin are main obstacles to hinder cellulose enzymolysis by cellulose enzyme [12]. Report views of scholars are combined according to comparison of acetone pretreatment and ethanol pretreatment data. It can be further concluded that lignin in the straw is the factor to affect cellulose enzyme enzymolysis.

The straw saccharification rate is very close after acetone-phosphoric acid pretreatment and ethanol-phosphoric acid pretreatment. It is obvious that ethanol also has the same ability of dissolving lignin as acetone. Since straw saccharification is very close after acetone and ethanol pretreatment, saccharification after straw pretreatment has the ultimate goal of fermentation and production of ethanol. The circulation chain of pretreatment, recycling and saccharification fermentation supplementing can be formed by organic solvent treatment method of replacing acetone with ethanol. It has high economic value to actual production.

\section{Influence of ethanol-ferric chloride pretreatment temperature on straw enzymatic} saccharification

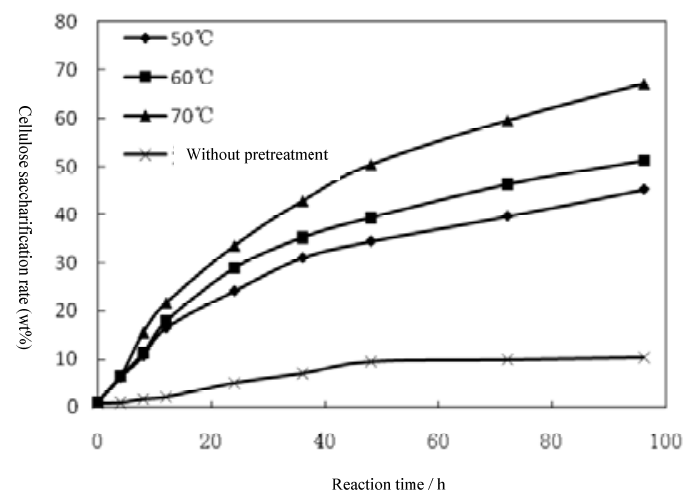

Figure 2 The effect of pretreatment temperature on rice straw saccharification

Figure 2 shows the change process of cellulose saccharification rate in straw with enzymolysis time after different pretreatment temperatures.

Figure 2 shows that pretreatment effect straw saccharification is the best at 70DEG $\mathrm{C}$. When the reaction lasts for 24h, 70DEG C pretreatment saccharification rate is $33.48 \%$, and pretreatment saccharification rate is $24.16 \%$ and $28.88 \%$ respectively at 50DEG $\mathrm{C}$ and 60DEG C; when the reaction lasts for $48 \mathrm{~h}$, the pretreatment saccharification rate is $50.43 \%$ at $70 \mathrm{DEG} \mathrm{C}$, pretreatment saccharification rate is $34.42 \%$ and $39.34 \%$ respectively at 50DEG $\mathrm{C}$ and 60DEG C; when the reaction lasts for $96 \mathrm{~h}$, the pretreatment saccharification rate is $67.06 \%, 51.37 \%$ and $45.19 \%$ respectively at 96DEG $\mathrm{C}$, 60DEG $\mathrm{C}$ and 50DEG $\mathrm{C}$. When ethanol-ferric chloride is used for pretreatment of straw, straw cellulose saccharification rate is increased with temperature increase, the temperature increase has prominent promotion to straw enzymatic saccharification rate because straw hemicellulose decomposition and lignin solution effect are strengthened with temperature increase, the cross-linked structure between the lignin and hemicellulose is damaged, thereby reducing straw crystallinity. Temperature has different influence on ethanol-ferric chloride and acetone-phosphoric acid pretreatment as well as ethanol-phosphoric acid pretreatment possibly because phosphoric acid with higher concentration can decompose straw cellulose with temperature increase. After the best enzymatic saccharification rates of straw after ethanol-ferric chloride pretreatment, acetone-phosphoric acid pretreatment and ethanol-phosphoric acid pretreatment are compared, the rates are $67.06 \%, 62.46 \%$ and $57.75 \%$ respectively. It can be concluded that the saccharification rate is highest after ethanol-ferric chloride pretreatment because temperature rise can promote ferric 
chloride to decompose hemicellulose and ethanol to dissolve lignin. In addition, ferric chloride also can accelerate lignin decomposition by proton catalyst.

Influence of ethanol-ferric chloride pretreatment time on straw enzymatic saccharification Figure 3 shows that saccharification rate change process of cellulose in straw with enzymolysis time after different pretreatment times.

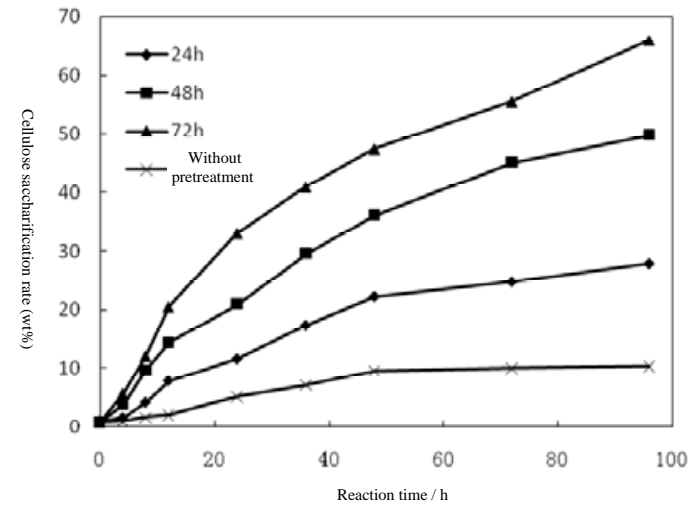

Figure 3 The effect of pretreatment time on rice straw saccharification

Figure 3 shows that enzymatic saccharification rate is the best after pretreatment for $72 \mathrm{~h}$. When the reaction lasts for $24 \mathrm{~h}$, pretreatment $72 \mathrm{~h}$ saccharification rate is $33.06 \%$. Pretreatment $24 \mathrm{~h}$ and $48 \mathrm{~h}$ saccharification rate is $11.61 \%$ and $20.92 \%$ respectively; when the reaction lasts for $48 \mathrm{~h}$, pretreatment $72 \mathrm{~h}$ saccharification rate is $47.39 \%$, pretreatment $24 \mathrm{~h}$ and $48 \mathrm{~h}$ saccharification rate is $22.85 \%$ and $36.20 \%$ respectively; when the reaction lasts for $96 \mathrm{~h}$, the pretreatment $72 \mathrm{~h}$, $48 \mathrm{~h}$ and $24 \mathrm{~h}$ saccharification rate is $65.91 \%, 49.81 \%$ and $27.93 \%$ respectively. Ethanol-ferric chloride pretreatment straw is used; enzymatic saccharification rate is higher with increase of pretreatment time because straw hemicellulose decomposition and lignin dissolution are promoted with increase of pretreatment time.

Comparison of the best saccharification rate between pretreatment time and temperature Figure 4 shows the influence of pretreatment time and temperature on saccharification rate of cellulose in straw.

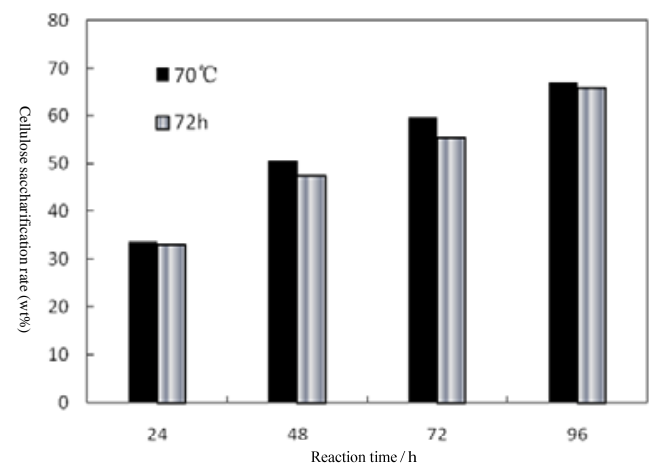

Figure 4 The comparison of rice straw saccharification between temperature and time

Figure 4 shows that when the reaction lasts for $24 \mathrm{~h}$, saccharification rate after pretreatment is $33.48 \%$ at $70 \mathrm{DEG} \mathrm{C}$, the saccharification rate is $33.06 \%$ after pretreatment time for $72 \mathrm{~h}$; when the reaction lasts for 72 hours, saccharification rate after pretreatment is 59.53\% at 70DEG C, the saccharification rate is $55.45 \%$ after pretreatment time for $72 \mathrm{~h}$. When the reaction lasts for $96 \mathrm{~h}$, saccharification rate after pretreatment is $67.06 \%$ at 70 DEG C. The saccharification rate is $65.91 \%$ after pretreatment time for $72 \mathrm{~h}$. The saccharification rate under the condition of 70DEG C and pretreatment for $1 \mathrm{~h}$ is not greatly different from the saccharification rate under the condition of room temperature and pretreatment for $72 \mathrm{~h}$. It is obvious that pretreatment time can be reduced due to temperature increase; temperature can be reduced by increasing pretreatment time, thereby reaching similar pretreatment effect. 


\section{Conclusion}

Straw saccharification is very close after acetone-phosphoric acid pretreatment and ethanol-phosphoric acid pretreatment. In addition, saccharification after straw pretreatment has final purpose that it is used for fermentation and production of ethanol. However, ethanol also can be used for pretreatment of straw, thereby forming cycle production. The circulation chain of pretreatment, recycling and saccharification fermentation supplementing can be formed by organic solvent treatment method of replacing acetone with ethanol with high economic value on actual production. After ethanol-ferric chloride organic solvent is adopted for pretreatment of straw, straw enzymatic saccharification rate can be increased effectively. The straw enzymatic saccharification after pretreatment can be increased from $10.03 \%$ without pretreatment to $65.91 \%$ under the condition of 70DEG C water bath heating and pretreatment time for $1 \mathrm{~h}$. Temperature can be increased to reduce the pretreatment time in ethanol-ferric chloride organic solvent pretreatment, pretreatment time should be increased for lowering temperature.

\section{References}

[1]Sun Y, Cheng J Y. Hydrolysis of lignocellulosic materials for ethanol production: a review. Bioresource Technology, 2002, 83(1): 1-11.

[3]Wingren A, Galbe M, Zacchi G. Techno-Economic Evaluation of Producing Ethanol from Softwood: Comparison of SSF and SHF and Identification of Bottlenecks. Biotechnology Progress, 2003, 19(4): 1109-1117.

[4] Ingram L O, Doran J B. Conversion of cellulosic materials to ethanol. FEMS Microbiol Reviews, 1995, 16(2-3): 235-241

[5] Linde M, Galbe M, Zacchi G. Bioethanol production from non-starch carbohydrate residues in process streams from a dry-mill ethanol plant. Bioresource Technology, 2008, 99(14): 6505-6511.

[6]Yawalata D. Catalytic selectivity in alcohol organosolv pulping of spruce wood. Canada: University of British Columbia, 2001.

[7] Lopez F, Alfaro A, Jimenez L, et al. Alcohols as organic solvents for the obtainment of cellulose pulp. Afinidad, 2006, 63(523):174-182.

[8] Holtzapple M T, Humphrey A E. The effect of organosolv pretreatment on the enzymic hydrolysis of poplar. Biotechnology Bioengineering, 1984, 26(7): 670-676.

[9] Liu Li, Sun Junshe , Li Min, et al. Enhanced enzymatic hydrolysis and structural features of corn stover by FeCl3 pretreatment. Bioresource Technology, 2009, 100(23): 5853-5858.

[10]Teramoto Y, Tanaka N, Lee S H, et al. Pretreatment of Eucalyptus Wood Chips for Enzymatic Saccharification Using Combined Sulfuric Acid-Free Ethanol Cooking and Ball Milling. Biotechnology and Bioengineering, 2008, 99(1): 75-85.

[11] Araque E, Parra C, Freer J, et al. Evaluation of organosolv pretreatment for the conversion of Pinus radiata D Don to ethanol. Enzyme and Microbial Technology, 2008, 43(2): 214-219.

[12] Sun F B, Chen H Z. Comparison of atmospheric aqueous glycerol and steam explosion pretreatments of wheat straw for enhanced enzymatic hydrolysis. Journal of Chemical Technology and Biotechnology, 2008, 83(5):707-714. 